\title{
Implementation and Outcomes of a Telehealth Neonatology Program in a Single Healthcare System
}

\author{
Lory J. Maddox ${ }^{1,2 *}$, Jordan Albritton ${ }^{1,3}$, Janice Morse ${ }^{2,4}$, Gwen Latendresse ${ }^{2}$, Paula Meek ${ }^{2}$ \\ and Stephen Minton ${ }^{5}$
}

${ }^{1}$ Intermountain Connect, Intermountain Healthcare, Salt Lake City, UT, United States, ${ }^{2}$ College of Nursing, University of Utah, Salt Lake City, UT, United States, ${ }^{3}$ RTI International, Research Triangle Park, Durham, NC, United States, ${ }^{4}$ University of Alberta, Edmonton, AB, Canada, ${ }^{5}$ Neonatal TeleHealth Intermountain Healthcare, Salt Lake City, UT, United States

OPEN ACCESS

Edited by:

Mark Lo,

University of Washington,

United States

Reviewed by:

Daniele Trevisanuto,

University Hospital of Padua, Italy

Naveed Hussain,

University of Connecticut Health

Center, United States

*Correspondence:

Lory J. Maddox

lory.maddox@imail.org

Specialty section: This article was submitted to

Neonatology,

a section of the journal

Frontiers in Pediatrics

Received: 31 December 2020 Accepted: 09 March 2021

Published: 23 April 2021

Citation:

Maddox LJ, Albritton J, Morse J, Latendresse G, Meek $P$ and Minton S (2021) Implementation and Outcomes of a Telehealth Neonatology Program in a Single Healthcare System.

Front. Pediatr. 9:648536. doi: 10.3389/fped.2021.648536
Background: Intermountain Healthcare, an early adopter and champion for newborn video-assisted resuscitation (VAR), identified a reduction in facility-level transfers and an estimated savings of $\$ 1.2$ million in potentially avoided transfers in a 2018 study. This study was conducted to increase understanding of VAR at the individual, newborn level.

Study Aim: To compare transfers to a newborn intensive care unit (NICU), length of stay (LOS), and days of life on oxygen between newborns managed by neonatal VAR and those receiving standard care (SC).

Methods: This retrospective, nonequivalent group study includes infants born in an Intermountain hospital between 2013 and 2017, 34 weeks gestation or greater, and requiring oxygen support in the first 15 minutes of life. Data came from billing and clinical records from Intermountain's enterprise data warehouse and chart reviews. We used logistic regression to estimate neonatal VAR's impact on transfers. Negative binomial regression estimated the impact on LOS and days of life on supplemental oxygen.

Results: The VAR intervention was used in 46.2 percent of post-implementation cases and is associated with (1) a 12 percentage points reduction in the transfer rate, $p=0.02$, (2) a reduction in spoke hospital $(\mathrm{SH})$ LOS of $8.33 \mathrm{~h}(p<0.01)$ for all transfers; (3) a reduction in SH LOS of $2.21 \mathrm{~h}(p<0.01)$ for newborns transferred within $24 \mathrm{~h}$; (4) a reduction in SH LOS of $17.85 \mathrm{~h}(p=0.06)$ among non-transferred newborns; (5) a reduction in days of life on supplemental oxygen of 1.4 days $(p=0.08)$ among all transferred newborns, and (6) a reduction in days of life on supplemental oxygen of 0.41 days $(p=0.04)$ among non-transferred newborns.

Conclusion: This study provides evidence that neonatal VAR improves care quality and increases local hospitals' capabilities to keep patients close to home. There is an ongoing demand for support to rural and community hospitals for urgent newborn resuscitations, and complex, mandatory NICU transfers. Efforts may be necessary to encourage neonatal VAR since the intervention was only used in 46.2 percent of this study's potential cases. Additional work is needed to understand the short- and long-term impacts of Neonatal VAR on health outcomes.

Keywords: telehealth, newborn, resuscitation, implementation, telemedicine, transfers, length of stay, video-assisted resuscitation 


\section{INTRODUCTION}

Over 40 years ago, video technology was used to reduce newborn mortality and morbidity in high-risk maternal-newborn populations geographically separated from neonatologists (1). Over the past decade, the use and effectiveness of synchronous audio-video communications in pediatric care, newborn care, and support for newborn resuscitations has increased (2-4). Consumer demand, medical need, and federal reimbursement represent an acceptance of telemedicine and telehealth services (5-7). In the face of the overwhelming demand for telehealth services during the global pandemic, program evaluation becomes increasingly important despite the challenges of rapid cycle development, implementation, and success measures.

Telehealth video-assisted resuscitation (VAR) programs vary in implementation, and there is limited evidence of the impact of these programs. Three of the earliest VAR programs began in 2013. Randall Children's Hospital supported five low-risk maternity centers and participated in about two percent of all births $(8,9)$. The Mayo clinic also began using telehealth technology to support six spoke sites (10). Intermountain Healthcare conducted its first neonatal video consult in 2013. By early 2016, it had deployed the neonatal video consult service to over 16 hospitals in the Intermountain West.

Early in the implementation, NICU hub neonatologists and spoke sites shared anecdotal stories of successful VAR, preventing transfers, and increasing confidence in their ability to conduct a newborn resuscitation. This study was informed from early implementation success stories, Intermountain and UC Davis studies on reduced transfer rates, and improved resuscitation quality reported by Randall Children's Hospital and the Mayo Clinic (9, 11-13). This study's primary aim was to determine the influence of a neonatologist VAR on transfers to a NICU, birth facility length of stay, This study's primary aim was to determine the influence of a neonatologist VAR on transfers to a newborn intensive care unit (NICU), birth facility length of stay, and days of life on supplemental oxygen.

\section{MATERIALS AND METHODS}

In 2013, Intermountain piloted an innovative program to provide neonatal VAR to remote hospitals in Southwest Utah 54 and 118 miles away from the hub neonatal intensive care unit (NICU) in St. George, Utah. Over the next 3 years, this program was expanded to four NICU hubs and 16 spoke sites in Utah and its immediate borders. The Utah neonatal VAR project developed technical solutions, assessed clinical feasibility, conducted the implementation, and evaluated operational and clinical solutions. Two individuals on this paper were part of the implementation team, LM as operations manager and SM as neonatal telemedicine medical director.

Telehealth systems often operate with a "hub and spoke" model. In the case of Intermountain's newborn VAR program, the neonatologist staffed tertiary NICUs as the hub providing care via telehealth to smaller regional or community hospitals, the spokes. The newborn and family receive in-person care at the spoke facility. A single NICU hub will support multiple spoke hospitals as part of their regionalized maternal-newborn care system (14). This manuscript will refer to the NICUs as hubs, and local nursery's as the spokes.

\section{Telehealth Equipment}

Design considerations for the neonatal environment include the diversity of newborn warmers, incubator designs, and the limited space around a warmer-approximately 48 inches deep and 25 inches wide. No telehealth equipment could be permanently attached to newborn warmers since warmers are FDA-regulated devices and are frequently moved throughout nurseries and hospitals.

Telehealth equipment was internally developed by the Intermountain telehealth technology team using currently available technology. The telehealth equipment included a palm-size Axis pan-tilt-zoom camera, a dedicated computer, and a monitor secured to the newborn area headwall or used as a mobile telehealth workstation (Figure 1). Microsoft video conferencing applications were customized to allow room selection, remote audio-video controls, and role-based access. Synchronous audio-video consults were conducted on Intermountain's intranet and approved by compliance and information systems security teams.

Each NICU hub had at least two telehealth-enabled workstations. Hardwired synchronous audio-video conferencing equipment was installed in delivery rooms, cesarean section operating rooms, and nursery locations in spoke hospitals. A mobile solution involved a palm-sized Axis camera, a Dell All-In-One computer, and Intermountain's customized video conferencing software. This allowed clinicians at spoke sites to access neonatologists for telehealth consults for at-risk neonates anywhere in their facility. Once the neonatologist was notified about the baby's location, the neonatologist could initiate a video connection, remotely control the camera using pan-tilt-zoom features, and adjust the audio for the spoke site hospital and themselves. These design features allowed the spoke site clinicians to focus on the newborn resuscitation, not the telehealth technology solution.

\section{Clinical Usability}

Telehealth technologies were used to help spoke sites with simulation training, consults, and VAR, developing an early version of newborn resuscitation telemedicine program (NRTP) $(5,14-17)$. Clinical staff at spoke sites were asked to notify the neonatologists as early as possible, often before delivery, to allow time to establish a video connection. Indications for early notification for neonatologists were drawn from obstetrical high-risk categorization for mothers and fetuses $(17,18)$. Early notification provided time for neonatologists at the hub site to establish a video connection with the spoke site, discuss, and prepare clinical staff for the neonate's birth just as they would in an in-person delivery.

Systemwide implementation began after clinical feasibility, standardized telehealth equipment, and workflows had been established. At the end of 2016, there were 126 newborn 


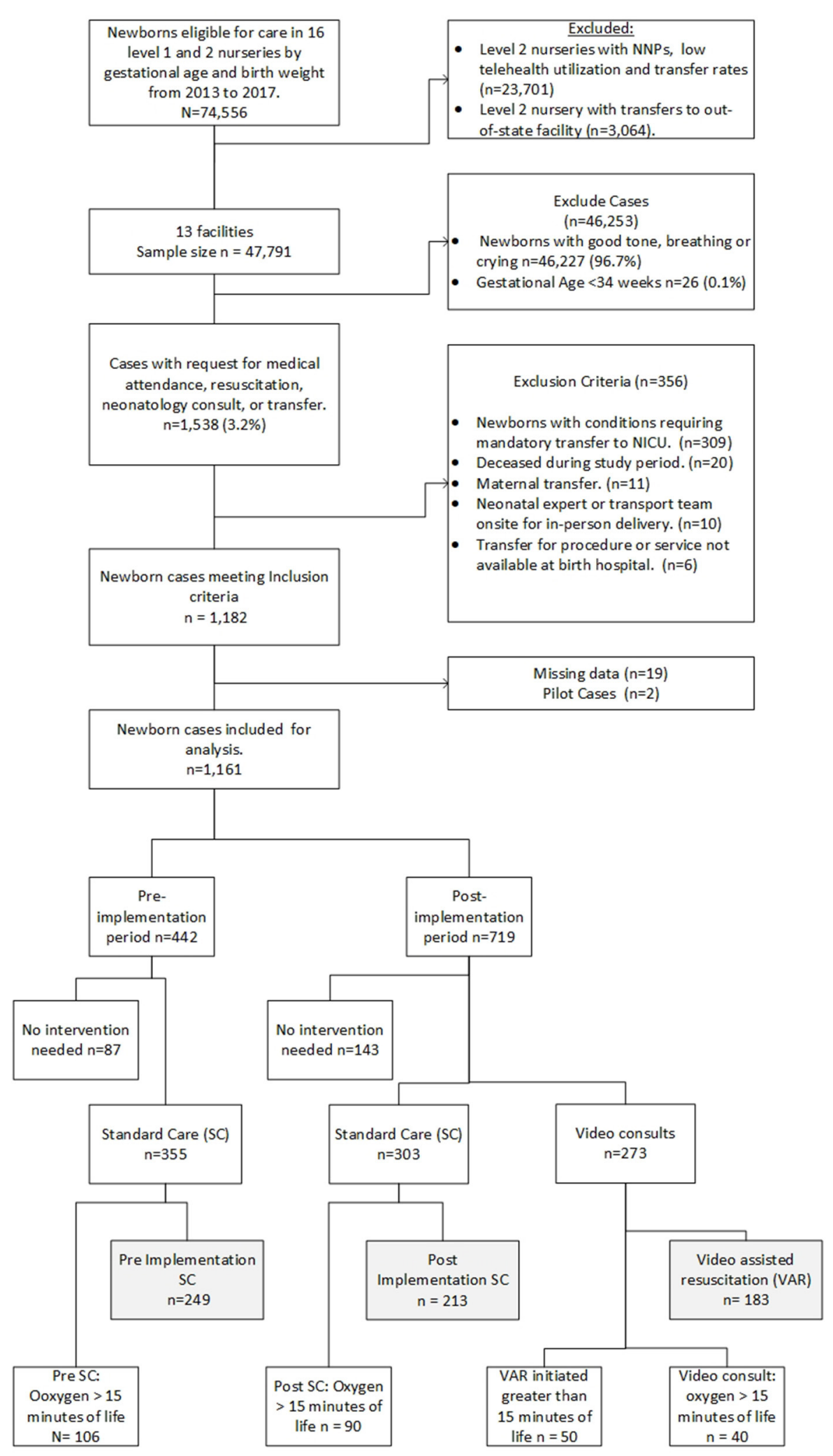

FIGURE 1 | Sample collection. 
telehealth in-room solutions and 14 mobile carts for newborn VAR; consults were being conducted at 16 spoke site locations. Hub site NICUs had at least two telehealth workstations to ensure that neonatologists had easy access to support emergent events.

\section{STUDY DESIGN}

In this retrospective, non-equivalent, pre-post telehealth implementation study, we analyzed a subset of newborns with no mandatory previously determined transport requirements. The study sample includes newborns with a gestational age of at least 34 weeks with oxygen administered within the first 15 min of life, born between 2013 and 2017. The newborns had a telephone or video neonatology consult or were transferred to a tertiary and quaternary NICU. Records obtained from the enterprise data warehouse (EDW) did not always include a scanned document indicating "resuscitation," so manual chart review was completed. Chart reviews included minutes of life to oxygen administration, VAR or standard care, and days of life on supplemental oxygen.

Initiation of oxygen, resuscitation measures, or transfers were based upon medical necessity. These are clinical decisions not based on parental concern or ability to pay for services. Therefore, randomization to a control group is not possible. Intermountain Healthcare and the University of Utah Institutional Review Board approved this study.

\section{Data Collection and Analysis}

Three groups were identified during data abstraction and initial analysis. Group $1(n=183)$ includes VAR conducted by the neonatologist after program implementation. Group $2(n=213)$ consisted of post-implementation standard care (SC) when neonatology consults were via telephone. Group 3 $(n=249)$, consisted of SC pre-implementation period. Two of the 16 spoke sites were excluded due to low telehealth and transfer rates. A third spoke site was excluded because newborns were transferred to an out-of-state NICU where follow-up chart review is not possible. Newborn diagnoses codes were used to exclude newborns with conditions requiring a mandatory transport to a quaternary or tertiary NICU. Additional exclusion criteria include newborns deceased during the study period, newborns transferred for maternal transports, newborns managed by in-person neonatology advanced practice practitioners, neonatologists, or transport team present at delivery, or mandatory transfers for service or procedure not available at the birthing hospital (Figure 2). Exclusion criteria were applied to generate a more homogenous population for this study. Additionally, we excluded cases $(n=50)$ where the VAR began more than $15 \mathrm{~min}$ after birth; in these cases, it was decided that it was unlikely that the neonatologist would have a significant impact on the resuscitation event. We conducted a sensitivity analysis to test the results and included cases with VAR $>15$ min of life.

All newborns were born in an Intermountain facility. Therefore, data from maternal and neonatal billing codes, diagnoses, clinical event data, and newborn gestational age, weight, gender, and Apgar scores were available to the researchers from the electronic medical record (EMR) and the EDW. Data on type of neonatology consult, minutes of life oxygen administered, and days of life on supplemental oxygen were abstracted from chart reviews, recorded in REDCap, and stored in a passwordprotected database. Consult types were determined from billing data and verified during chart review. Transfers and LOS data was determined from clinical events recorded in the EDW. Data discrepancies between billing data and chart review were reconciled after reviewing with the research team. A master data set combined all data sources.

\section{Statistical Analysis}

We summarized characteristics for newborns with oxygen initiated in the first $15 \mathrm{~min}$ of life $(n=645)$ and the maternal and newborn diagnoses for the study population. All $t$-tests and $p$ values are bivariate and presented for informational purposes only. There was no statistical difference in gestational age, weight, and gender for the newborns between the study groups. All newborns in this study had a 1-min Apgar score of $<7$. The mean 1-min Apgar score was 4.1 with a standard deviation of 2.4 in the VAR group and was significantly lower than the postimplementation SC group (mean $=5.0$, SD 2.6), $p<0.001$, and pre-implementation SC group (mean $=4.7, \mathrm{SD}=2.9) p=0.03$ (Table 1 Newborn sample characteristics and Table 2 Maternal and newborn diagnoses).

We used logistic regression to identify factors associated with a neonatology consult (Table 3 Factors associated with neonatology consult before or within $15 \mathrm{~min}$ of birth). In this study, it was vital to parse neonatology VAR's effect, the independent variable, from the pre-and post-implementation period and confounding variables. Thus, in the main analyses, we controlled for factors significantly associated with early notification and other variables deemed important based on clinical expertise. Control variables included time (pre or post-implementation period, newborn gestational age, gender, multiple gestation, 1-min Apgar scores, maternal chorioamnionitis, pregnancy-induced hypertension, eclampsia, pre-eclampsia, hemorrhage, intrapartum abnormal fetal heart tones, newborn meconium, umbilical cord or placenta complications, and nursery level. All statistical tests are conducting with Microsoft ExcelPro 16.0 and Stata 15.1.

We used regression analyses to evaluate the impact of VAR on key outcomes. Because of the emphasis on the first few minutes, hours, and days of life, the data collected to evaluate clinical outcomes are not normally distributed. We used logistic regression to estimate the impact of VAR on transfer rates. Count variables were overdispersed, meaning the variance in the data is greater than the mean. Thus, we used negative linear regression to determine the neonatology VAR program's effects on newborn LOS and days of life on supplemental oxygen.

A pre-study statistical power analysis was performed for sample size estimation. The power analysis was based on data from an internal pilot study comparing the overall length of stay rates between pre-and post-implementation of the VAR program. The pilot study's effect size was 0.19 , considered a small effect size, and was based on nursery level and newborns' gestational age but did not account for maternal or newborn risk factors. The sample 


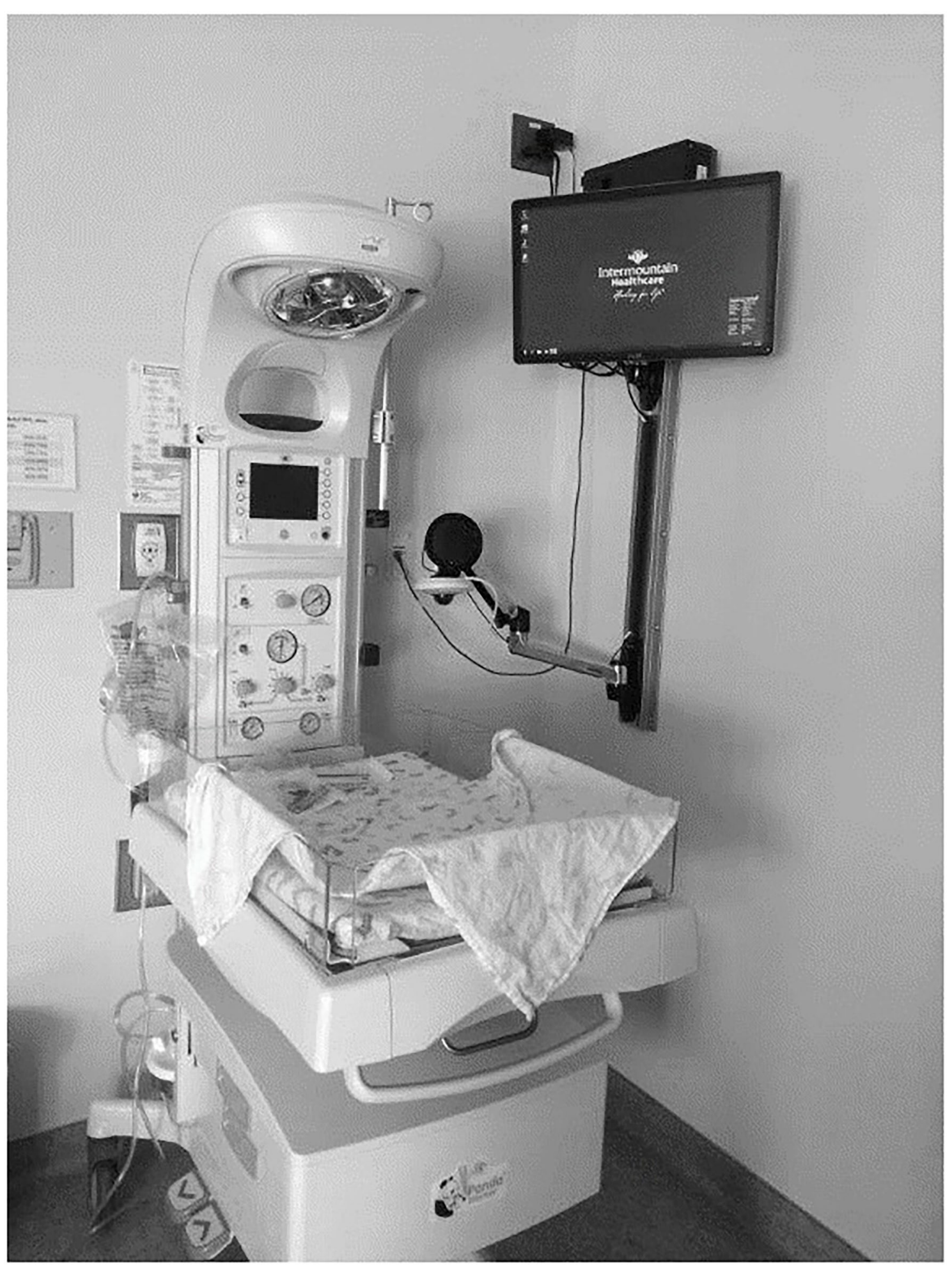

FIGURE 2 | Wall mounted newborn telehealth station at spoke site. 
TABLE 1 | Newborn sample characteristics.

\begin{tabular}{|c|c|c|c|c|c|}
\hline & \multicolumn{3}{|c|}{ Post-implementation } & \multicolumn{2}{|c|}{ Pre-implementation } \\
\hline & $\begin{array}{l}\text { Video assisted resuscitation } \\
\qquad(n=183)\end{array}$ & $\begin{array}{l}\text { Standard care } \\
\qquad(n=213)\end{array}$ & $\boldsymbol{P}$ & $\begin{array}{l}\text { Standard care } \\
\qquad(n=249)\end{array}$ & $\boldsymbol{P}$ \\
\hline Gestational age, mean (SD) & $38.2(2.01)$ & $38.1(2.0)$ & 0.58 & $38.4(1.8)$ & 0.40 \\
\hline Gestational age category, $\boldsymbol{n}(\%)^{\mathrm{A}}$ & & & 0.36 & & 0.07 \\
\hline 34 0/7-35 6/7 weeks & $32(17.5)$ & $40(15.2)$ & & $26(10.4)$ & \\
\hline 36 0/7-37 6/7 weeks & $39(21.3)$ & $73(27.8)$ & & $75(30.1)$ & \\
\hline 38 0/7-39 6/7 weeks & $73(39.9)$ & $90(34.2)$ & & 95 (38.2) & \\
\hline 40 weeks or greater & $39(21.3)$ & $60(22.8)$ & & $53(21.3)$ & \\
\hline \multicolumn{6}{|l|}{ Birth weight (grams) } \\
\hline Mean (SD) & $3,145(599)$ & $3,116(587)$ & 0.62 & $3,211(510)$ & 0.23 \\
\hline Gender & & & 0.51 & & 0.11 \\
\hline Female & $62(33.9)$ & $97(36.9)$ & & $103(41.4)$ & \\
\hline Male & $121(66.1)$ & $166(63.1)$ & & 146 (58.6) & \\
\hline \multicolumn{6}{|l|}{ APGAR, mean (SD) } \\
\hline $1 \min ^{B}$ & $4.1(2.4)$ & $5.0(2.6)$ & $<0.001$ & $4.7(2.9)$ & 0.03 \\
\hline $5 \min ^{B}$ & $6.7(1.8)$ & $6.8(1.9)$ & 0.75 & $6.7(2.2)$ & 0.99 \\
\hline $10 \min ^{\mathrm{C}}$ & $7.4(1.4)$ & $7.4(1.6)$ & 0.64 & $7.3(2.0)$ & 0.85 \\
\hline
\end{tabular}

Results from t-tests with the assumption of unequal variances unless otherwise specified.

${ }^{A}$ Fishers exact test for categorical variables.

${ }^{B}$ VC, $n=180$, Pre-UC, $n=246$.

${ }^{C}$ VC, $n=74$, Post-UC, $n=95$, Pre-UC $n=132$.

size for this study was determined to be 565 cases with an alpha $=0.05$, power $=0.80$, a two-sided t-test.

\section{RESULTS}

In the logistic regression model, the VAR group had a significant decrease in the transfer rate of 12 percentage points, $p=0.02$, $\mathrm{SE}=0.05$. For all transfers, the neonatology VAR intervention was associated with decreased LOS of $8.33 \mathrm{~h}, p<0.001, \mathrm{SE}=1.3$. For newborns remaining at the spoke facility, VAR was associated with a LOS reduction of $17.9 \mathrm{~h}, p=0.06, \mathrm{SE}=9.5$. For newborns transferred within $24 \mathrm{~h}$, VAR was associated with a reduced LOS by $2.21 \mathrm{~h}, p<0.01$, SE $=0.60$.

Neonatal VAR influenced days of life on supplemental oxygen. For newborns transferred to a NICU, neonatal VAR was associated with a reduction in days of life on supplemental oxygen by 1.41 days, $p=0.08, \mathrm{SE}=0.80$. Newborns that were not transferred spent an average of $9.84 \mathrm{~h}$ less (0.41 days), $p=0.04$, $\mathrm{SE}=0.20$, than the standard care groups

(Table 4 VAR influence on transfers, birth facility length of stay, and days on supplemental oxygen).

The sensitivity analysis, including 50 additional cases with VAR conducted later than 15-min of life, produced similar results to those just reported. The VAR group transfer rate increased to 14 percentage points, $p<0.01, \mathrm{SE}=0.04$. For all transfers, the neonatology VAR intervention was associated with decreased LOS of $7.03 \mathrm{~h}, p<0.001, \mathrm{SE}=1.24$. For newborns remaining at the spoke facility, VAR was associated with a LOS reduction of $16.71 \mathrm{~h}, p=0.07, \mathrm{SE}=9.05$. For newborns transferred within $24 \mathrm{~h}, \mathrm{VAR}$ was associated with a reduced LOS by $1.74 \mathrm{~h}, p<0.01$, $\mathrm{SE}=0.58$.
For newborns transferred to a NICU, neonatal VAR was associated with reduced days of life on supplemental oxygen by 1.39 days, $p=0.76, \mathrm{SE}=0.07$. There was no change in days of life on supplemental oxygen for newborns remaining at the spoke site.

\section{DISCUSSION}

Intermountain Healthcare's neonatology service was an early adopter and champion for VAR. This program was implemented to provide expert support for high-risk births and post-delivery care to reduce unnecessary transfers (15). Guidelines to request additional medical assistance from an on-call pediatrician or another qualified medical provider were in place before the neonatology VAR program. During program implementation, spoke sites were encouraged to follow existing guidelines to request on-call medical attendance. Once the on-call provider was notified, the neonatologists would be called for an anticipated VAR. Frequently, the neonatology video consult would be established before the in-person medical provider's arrival. Establishing a video connection before birth allowed the neonatologist to receive a report, anticipate clinical scenarios, review resuscitation protocols, and emergency resuscitation equipment with the spoke site team $(5,15,19)$.

In this study, acute maternal diagnoses of chorioamnionitis, pregnancy-induced hypertension, eclampsia, pre-eclampsia, hemorrhage, intrapartum abnormal fetal heart tones, and fetal meconium, umbilical cord, or placenta complications were most frequently associated with a request for a neonatology VAR before or within $15 \mathrm{~min}$ of birth. An acute maternal, intrapartum, or fetal event's urgency may explain the higher frequency of these 
TABLE 2 | Maternal and newborn diagnoses.

\begin{tabular}{ccc}
\multicolumn{3}{c}{ Post-implementation } \\
\hline VAR & SC & $P$ \\
$(n=183)$ & $(n=213)$ &
\end{tabular}

\section{Maternal diagnoses, $\boldsymbol{n}$ (\%)}

Chorioamnionitis

Infection

Hypertension, Pregnancy induced, Pre-eclampsia, eclampsia

Mood and anxiety disorders

Diabetes mellitus (gestational, type 1 and 2)

Metabolic disorders other than diabetes

Anemia or blood disorders

Obesity

Respiratory Disorders

Substance Abuse

Uterine bleeding, complications of labor

Fetal diagnosis, $\boldsymbol{n}$ (\%)

Multiple Gestation

Small for dates

Large for dates

Polyhydramnios

Oliogohydramnios

\section{Intrapartum diagnosis, $\boldsymbol{n}$ (\%)}

Abnormal fetal heart tracings

Abnormal presentation

Nuchal cord

Meconium associated with birth

Umbilical cord complications

Instrumental delivery

Placenta Previa, abruption, hemorrhage

General anesthesia

Narcotic use within four hours of delivery

Newborn diagnosis, $\boldsymbol{n}(\%)$

Respiratory

Sepsis, actual, and rule-out

Fluid, electrolyte, and metabolic imbalances

Hypoxia

Pneumothorax

Cardiovascular disorders - other than congenital

Emphysema

Abnormal movements, seizure assessment

Pneumonia

Hypoglycemia

Hypovolemia

\begin{tabular}{|c|c|c|c|c|}
\hline $59(32.2)$ & 39 (18.3) & $<0.001$ & 58 (23.3) & 0.04 \\
\hline $24(13.1)$ & 26 (12.2) & 0.95 & $9(3.6)$ & $<0.001$ \\
\hline $45(24.6)$ & $30(14.1)$ & $<0.01$ & 42 (16.9) & 0.05 \\
\hline $24(13.1)$ & 34 (16.0) & 0.69 & $31(12.5)$ & 0.84 \\
\hline $17(9.3)$ & $30(14.1)$ & 0.56 & $29(11.7)$ & 0.05 \\
\hline $21(11.5)$ & 27 (12.7) & 0.82 & 95 (38.2) & $<0.001$ \\
\hline $17(9.3)$ & $13(6.1)$ & 0.28 & $21(8.4)$ & 0.76 \\
\hline $8(4.4)$ & $18(8.5)$ & 0.15 & $17(6.8)$ & 0.27 \\
\hline $6(3.3)$ & 12 (5.6) & 0.49 & $20(8.0)$ & 0.03 \\
\hline $5(2.7)$ & $9(4.2)$ & 0.30 & $5(2.0)$ & 0.63 \\
\hline $11(6.0)$ & $4(1.9)$ & 0.04 & $8(3.2)$ & 0.18 \\
\hline 27 (14.8) & $20(9.4)$ & 0.11 & $8(3.2)$ & $<0.001$ \\
\hline $13(7.1)$ & $22(10.3)$ & 0.25 & 68 (27.3) & $<0.001$ \\
\hline $14(7.7)$ & $15(7.0)$ & 0.82 & $6(2.4)$ & 0.09 \\
\hline $7(3.8)$ & $7(3.3)$ & 0.81 & $4(1.6)$ & 0.30 \\
\hline $1(0.6)$ & $4(1.9)$ & 0.22 & $5(2.0)$ & 0.38 \\
\hline 67 (36.6) & 53 (24.9) & 0.01 & 66 (26.5) & 0.03 \\
\hline 57 (31.2) & 46 (21.6) & 0.03 & 42 (16.9) & 0.00 \\
\hline $50(27.3)$ & 44 (20.7) & 0.12 & $54(21.7)$ & 0.18 \\
\hline 40 (21.9) & 28 (13.2) & 0.02 & 43 (17.3) & 0.24 \\
\hline $18(9.8)$ & $17(8.0)$ & 0.52 & $59(23.7)$ & 0.00 \\
\hline $8(4.4)$ & $6(2.8)$ & 0.41 & $12(4.8)$ & 0.83 \\
\hline $16(8.7)$ & $8(3.8)$ & 0.04 & $16(6.4)$ & 0.38 \\
\hline $1(0.6)$ & $2(0.9)$ & 0.65 & $1(0.4)$ & 0.83 \\
\hline $1(0.6)$ & $3(1.4)$ & 0.38 & $3(1.2)$ & 0.46 \\
\hline $146(79.8)$ & 166 (77.9) & 0.65 & $181(72.7)$ & 0.09 \\
\hline 71 (38.8) & 66 (31.0) & 0.11 & 71 (28.5) & 0.03 \\
\hline $58(31.7)$ & $59(27.7)$ & 0.39 & 63 (25.3) & 0.15 \\
\hline $46(25.1)$ & $57(26.8)$ & 0.71 & $50(20.1)$ & 0.22 \\
\hline $14(7.7)$ & $13(6.1)$ & 0.54 & 34 (13.7) & 0.04 \\
\hline $17(9.3)$ & $18(8.5)$ & 0.77 & $36(14.5)$ & 0.09 \\
\hline- & $2(0.9)$ & 0.15 & 34 (13.7) & $<0.001$ \\
\hline $6(3.3)$ & $9(4.2)$ & 0.62 & 31 (12.4) & 0.001 \\
\hline $7(3.8)$ & $13(6.1)$ & 0.30 & $22(8.8)$ & 0.03 \\
\hline $18(9.8)$ & $24(11.3)$ & 0.64 & $20(8.0)$ & 0.52 \\
\hline 22 (12.0) & $17(8.0)$ & 0.19 & $14(5.6)$ & 0.02 \\
\hline
\end{tabular}

diagnoses in the VAR group. Ideally, these mothers would be transferred before birth to a regional maternity center equipped to manage these high-risk patients. However, maternal transfers are not possible when mothers with these conditions present to community and rural hospitals in advanced labor. Neonatal VAR acts as a safety net for these high-risk newborns by providing similar standards of care as the NICU hub $(15,20)$. Chronic maternal conditions such as hypertension, diabetes, or mood and anxiety disorders were higher in the post-implementation, standard care group. Pediatricians and family practice clinicians may feel more comfortable managing these patients without neonatal expert support at birth.

\section{Outcomes}

Transfers, spoke site LOS, and days on supplemental oxygen were used as outcome measures to evaluate this neonatal VAR 
TABLE 3 | Control factors for regression analyses.

\begin{tabular}{|c|c|c|c|c|c|}
\hline & \multirow[t]{2}{*}{ AMEA $^{A}$} & \multirow[t]{2}{*}{$\mathrm{SE}^{\mathrm{B}}$} & \multicolumn{2}{|c|}{$95 \% \mathrm{Cl}$} & \multirow[t]{2}{*}{$\boldsymbol{P}$} \\
\hline & & & LL & UL & \\
\hline Post implementation (Time period) & 0.48 & 0.03 & 3.38 & 5.48 & $<0.001$ \\
\hline \multicolumn{6}{|l|}{ Maternal factors } \\
\hline Chorioamnionitis & 0.11 & 0.04 & 0.29 & 1.29 & $<0.01$ \\
\hline Infection & -0.03 & 0.33 & -0.69 & 0.63 & 0.93 \\
\hline Hypertension, Pregnancy-induced, Pre-eclampsia, eclampsia & 0.11 & 0.04 & 0.23 & 1.34 & $<0.01$ \\
\hline Uterine bleeding, complications of labor & 0.97 & 0.63 & -0.25 & 2.20 & 0.12 \\
\hline \multicolumn{6}{|l|}{ Fetal factors } \\
\hline Multiple gestation & 0.11 & 0.05 & 0.10 & 1.46 & 0.02 \\
\hline \multicolumn{6}{|l|}{ Intrapartum factors } \\
\hline Abnormal fetal heart tracing & 0.05 & 0.03 & -0.14 & 0.79 & 0.18 \\
\hline Meconium associated with birth & 0.06 & 0.04 & -0.17 & 0.99 & 0.17 \\
\hline Umbilical cord complications & 0.06 & 0.05 & -0.28 & 1.13 & 0.23 \\
\hline Placenta Previa, abruption, hemorrhage & 0.09 & 0.06 & -0.24 & 1.57 & 0.14 \\
\hline \multicolumn{6}{|l|}{ Newborn factors } \\
\hline Gestational age & 0.01 & 0.01 & -0.08 & 0.18 & 0.47 \\
\hline Apgar, 1 min & -0.02 & 0.01 & -0.24 & -0.06 & $<0.01$ \\
\hline
\end{tabular}

Number of observations $=639$.

${ }^{A}$ Average marginal effects.

${ }^{B}$ Standard error.

TABLE 4 | VAR influence on transfers, birth facility length of stay, and days on supplemental oxygen.

\begin{tabular}{|c|c|c|c|c|}
\hline Outcome metrics & Number of observations & AME & SE & $\boldsymbol{P}$ \\
\hline Percentage point reduction in transfers ${ }^{\star}$ & 639 & -0.12 & 0.05 & 0.02 \\
\hline Reduced LOS in hours for all transferred newborns & 311 & -8.33 & 1.33 & $<0.01$ \\
\hline Reduced LOS in hours for newborns remaining at the birthing facility & 328 & -17.85 & 9.47 & 0.06 \\
\hline Reduced LOS in hours for newborns transferred within $24 \mathrm{~h}$ & 273 & -2.21 & 0.60 & $<0.01$ \\
\hline Reduced days on supplemental oxygen, transferred & 216 & -1.41 & 0.80 & 0.08 \\
\hline Reduced days on supplemental oxygen, not transferred & 303 & -0.41 & 0.20 & 0.04 \\
\hline
\end{tabular}

Linear regression was used to assess the effect of the VAR intervention on these outcomes unless noted otherwise.

All models used the same control factors listed in Table 3.

*Logistic regression analysis was used to determine transfer rates.

program (19). Direct measurements of resuscitation quality were not available in the newborn record. We identified a relatively homogenous group of newborns and chose transfers, LOS, and days on supplemental oxygen outcomes as indirect measures of the influence of VAR on resuscitation quality. This study is one of the earliest to report LOS and days on supplemental oxygen for neonatal VAR interventions at spoke sites.

In this study, neonatal experts were called to assist with the most acute maternal, fetal, and newborn conditions. Although higher-risk newborns are represented in the VAR group, VAR is associated with fewer transfers and supports prior studies that telehealth consults are associated with reduced transfer rates $(11,13)$. Additionally, VAR newborns transferred within the first $24 \mathrm{~h}$ of life had a LOS reduction of $2.21 \mathrm{~h}$. A benefit of telehealth is that patients can be more efficiently triaged to the appropriate level of care when specialists are involved with their care $(15,21)$.

Reductions in days of life on supplemental oxygen were also associated with VAR. Results from a simulation study using video consults for pre-transport evaluation found that neonatologists used less invasive respiratory support, i.e., continuous positive airway pressure (CPAP) vs. intubation, for transport (22). Neonatologists' tendency to use noninvasive ventilation techniques may contribute to fewer days on supplemental oxygen in the VAR group. Neonatology support for pre-transport stabilization and preference for CPAP vs. intubation for mild to moderate respiratory distress may also account for shorter LOS at spoke sites.

The sensitivity analysis we conducted suggests that neonatal VAR gains are the most beneficial when the VAR occurs at birth or within the first $15 \mathrm{~min}$ of life. When the neonatal expert is waiting for birth, they can receive a report and prepare bedside teams for a high-risk birth. The benefits of an early VAR intervention and the opportunity for "just in time" education offset the 20 percent of all medical attendance requests at birth that did not require NRP interventions.

The informal training spoke sites receive from ongoing communication and relationships with neonatal experts helps build knowledge and skills acquired during NRP certification. 
These ongoing synchronous audio-video interactions build upon existing telehealth-based simulation education and NRTP to improve clinical outcomes $(11,23-25)$. We were encouraged that our VAR rate was 46.2 percent, higher than the expected 34.5 percent rate reported by Fang et al. (26), reporting that 65.5 percent of users "did not use service because they did not have a clinical need." This study was not designed to explain why the spoke site chose (1) not to contact a neontologist for a VAR, (2) delay the consult until a transfer was required, or (3) why the NICU hub and spoke sites used the telephone. These questions deserve future study, especially in the post COVID19 period, when telehealth is the only plausible alternative to in-person care. The rapid adoption and implementation of telehealth during the COVID-19 pandemic and improved outcomes demonstrated in this study may persuade medical providers to increase telemedicine usage (27).

Paradoxically, the combination of increased neonatal video consults and steady transfer rates in the UC group led to an overall increase in neonatal consults in the postimplementation period. When establishing a neonatal VC program for newborn resuscitation and initial stabilization, there may be a period of increased neonatology workload. A telehealth service introduces new technology, workflows, and uncertain demand. Sample selection for this study provides a guideline for estimating demand for future neonatal video consult programs. Considerations for estimating the frequency of neonatal VC include:

1. Pre-transport stabilization cases for all premature and required newborn transfers.

2. Cases in which medical attendance at birth was requested or newborn resuscitation measures were performed.

3. Current transfer rates.

Estimates for neonatal video consult programs should allow for a period of technology deployment, testing, education, and early program adoption when both standard care transfers and VAR overlap.

\section{Limitations and Future Directions}

This study's retrospective design limits this study with sample selection from a single healthcare system and newborns $>34$ weeks gestation requiring oxygen within the first $15 \mathrm{~min}$ of life. Differences in spoke sites were controlled by nursery level, not by the implementation date. Since implementation occurred over a 16-month timeline, early spoke sites had more time to use telehealth during this study period. We used regression modeling to control for confounding variables associated with non-randomized studies.

Due to technology limitations and retrospective chart review, it was not feasible to determine each video consult's length of time. We may be missing data in the pre-implementation period since neonatology consults were not always documented. In some instances, resuscitation events were reconstructed during chart review. VAR within 15 min of life was chosen as the cutoff period, with approximately 80 percent of all VAR occurring before or within the first $15 \mathrm{~min}$ of life. We recognize that there may not be a difference between a VAR at 14 or $16 \mathrm{~min}$ but had to establish the study population. Newborns with a VAR $>15$ min of life were not included in this study. However, they were included in the sensitivity analysis which produced similar results.

Randomized controlled trials are challenging in real-world clinical settings, especially when an intervention, VAR, reduces transfers, facilitates timely triage, reduces LOS, and reduces days on supplemental oxygen. When and where VAR is available, we must ensure equitable access to high-quality neonatology care regardless of geographical location (15). Future studies should include prospective, observational, and ethnographic studies that emphasize decision-making to activate neonatology support in the delivery room. Team building and communication skills are critical areas of decision-making and can be studied in a simulated or clinical setting. Telehealth fundamentally changes communication styles and perceptions when the specialist is visible to the entire spoke site team, parents, and loved ones in the delivery room. Video recording of newborn resuscitation events affords clinicians the opportunity for an objective review of their performance like an elite athletes' review of their performance and provides an opportunity for coaching. In addition to using outcomes for quality improvement, transfer, LOS, and days of life on supplemental oxygen can be quantified for payers, hospitals, patients, and communities to describe a comprehensive neonatal VAR and NRTP valuation.

\section{CONCLUSION}

Improvements in care processes and outcomes provide evidence that neonatal VAR improves care quality. Neonatal VAR also helps increase the capabilities of local hospitals and keeps patients in their communities. There is an ongoing demand for support to rural and community hospitals for urgent newborn resuscitation, and complex, mandatory NICU transfers. Still, efforts may be necessary to encourage the use of neonatal VAR as the intervention was only used in 46.2 percent of potential cases in this study. Additional work is needed to understand the short- and long-term impacts of Neonatal VAR on health outcomes.

\section{DATA AVAILABILITY STATEMENT}

The raw data supporting the conclusions of this article will be made available by the authors, without undue reservation.

\section{ETHICS STATEMENT}

The studies involving human participants were reviewed and approved by Intermountain Healthcare and the University of Utah. Written informed consent from the participants' legal guardian/next of kin was not required to participate in this study in accordance with the national legislation and the institutional requirements.

\section{AUTHOR CONTRIBUTIONS}

LM, JA, JM, and SM conceived this study. LM and JA obtained and analyzed the data and contributed equally to 
this work. GL, PM, and JM supervised this study and share senior authorship. SM is last author and medical director for this program. All authors read, edited, and approved the final manuscript.

\section{FUNDING}

Partial funding provided to LM by Intermountain Healthcare educational program and nursing research grant.

\section{REFERENCES}

1. Jones PK, Halliday HL, Jones SL. Prediction of neonatal death or need for interhospital transfer by prenatal risk characteristics of mother. Med Care. (1979) 17:796-806. doi: 10.1097/00005650-197908000-00002

2. McCauley K, Kreofsky BL, Suhr BL, Fang JL. Developing a newborn resuscitation telemedicine program: a follow-up study comparing two technologies. Telemed e-Health. (2019) 26:589-96. doi: 10.1089/tmj.201 8.0319

3. Nadar M, Jouvet P, Tucci M, Toledano B. Impact of synchronous telemedicine models on clinical outcomes in pediatric acute care settings: a systematic review. Pediatr Crit Care Med. (2018) 19:e662-71. doi: 10.1097/PCC.0000000000001733

4. Tomines A. Pediatric telehealth: approaches by specialty and implications for general pediatric care. Adv Pediatr. (2019) 66:55-85. doi: 10.1016/j.yapd.2019.04.005

5. Donohue LT, Hoffman KR, Marcin JP. Use of telemedicine to improve neonatal resuscitation. Children. (2019) 6:E50. doi: 10.3390/children6040050

6. Fang J. Telemedicine for Newborn Resuscitation. (2017). doi: 10.1542/peds.141.1_MeetingAbstract.520

7. Sauers-Ford HS, Marcin JP, Underwood MA, Kim JH, Nicolau Y, Uy C, et al. The use of telemedicine to address disparities in access to specialist care for neonates. Telemed e-Health. (2018) 25:775-80. doi: 10.1089/tmj.201 8.0095

8. Scheans P. Telemedicine for neonatal resuscitation. Neonatal Netw. (2014) 33:283-7. doi: 10.1891/0730-0832.33.5.283

9. Scheans P, McElwain L. Telemedicine for neonatal resuscitation. J Obstetr Gynecol Neonatal Nurs. (2015) 44:S23. doi: 10.1111/1552-6909.12696

10. Fang JL, Collura CA, Johnson RV, Asay GF, Carey WA, Derleth DP, et al. Emergency video telemedicine consultation for newborn resuscitations: the mayo clinic experience. Mayo Clin Proc. (2016) 91:173543. doi: 10.1016/j.mayocp.2016.08.006

11. Albritton L, Maddox L, Dalto J, Ridout E, Minton S. The effect of a newborn telehealth program on transfers avoided: a multiple-baseline study. Health Aff. (2018) 37:1990-6. doi: 10.1377/hlthaff.2018.05133

12. Colby CE, Fang JL, Carey WA. Remote video neonatal consultation: a system to improve neonatal quality, safety and efficiency. Resuscitation. (2014) 85:e29-30. doi: 10.1016/j.resuscitation.2013.11.003

13. Haynes SC, Dharmar M, Hill BC, Hoffman KR, Donohue LT, KuhnRiordon KM, et al. The impact of telemedicine on transfer rates of newborns at rural community hospitals. Acad Pediatr. (2020) 20:63641 doi: 10.1016/j.acap.2020.02.013

14. Toward Improving the Outcome of Pregnancy III. Dimes M.o, editor (2010).

15. Olson CA, McSwain SD, Curfman AL, Chuo J. The current pediatric telehealth landscape. Pediatrics. (2018) 141:e20172334. doi: 10.1542/peds.2017-2334

16. Leone TA. Using video to assess and improve patient safety during simulated and actual neonatal resuscitation. Semin

\section{ACKNOWLEDGMENTS}

This study and the research behind it would not have been possible without the expertise and support of the entire telehealth team at Intermountain Healthcare as well as mentoring by senior authors. This research was conducted to fulfill degree requirements and may appear in print as: Maddox, Lory J. (2020) An analysis of telehealth neonatology consults on neonate management in level 1 and 2 nurseries. [Dissertation]. [Salt Lake City (UT)]: University of Utah.

\section{Perinatol. (2019) 43:151179. doi: 10.1053/j.semperi.2019.} 08.008

17. American College Obstetricians and Gynecologists. Levels of maternal care. Am J Obstet Gynecol. (2015) 212:259-71. doi: 10.1016/j.ajog.2014. 12.030

18. American Academy of Pediatrics Committee on Fetus and Newborn. Levels of neonatal care. Pediatrics. (2012) 130:587-97. doi: 10.1542/peds.2012-1999

19. Makkar A, McCoy M, Hallford G, Escobedo M, Szyld E. A hybrid form of telemedicine: a unique way to extend intensive care service to neonates in medically underserved areas. Telemed J E Health. (2018) 24:71721. doi: $10.1089 / \mathrm{tmj} .2017 .0155$

20. Hoffman AM, Bushey JA, Ocheltree TW, Smith MD. Historical perspectives: telemedicine in neonatology. Neoreviews. (2019) 20:e113-23. doi: 10.1542/neo.20-3-e113

21. Marcin JP, Shaikh U, Steinhorn RH. Addressing health disparities in rural communities using telehealth. Pediatr Res. (2016) 79:169-76. doi: 10.1038/pr.2015.192

22. Umoren RA, Gray MM, Schooley N, Billimoria Z, Smith KM, Sawyer TL. Effect of video-based telemedicine on transport management of simulated newborns. Air Med J. (2018) 37:317-20. doi: 10.1016/j.amj.2018.05.007

23. Escobedo MB, Shah BA, Song C, Makkar A, Szyld E. Recent recommendations and emerging science in neonatal resuscitation. Pediatr Clin North Am. (2019) 66:309-20. doi: 10.1016/j.pcl.2018.12.002

24. Beck JA, Jensen JA, Putzier RF, Stubert LA, Stuart KD, Mohammed $\mathrm{H}$, et al. Developing a newborn resuscitation telemedicine program: a comparison of two technologies. Telemed J E Health. (2018) 24:4818. doi: $10.1089 / \mathrm{tmj} .2017 .0121$

25. Makkar A, Siatkowski RM, Szyld E, Ganguly A, Sekar K. Scope of telemedicine in neonatology. Chinese J Contempor Pediatr. (2020) 22:396-408.

26. Fang, J. L., Asiedu, G. B., Harris, A. M., Carroll, K., and Colby, C. E. A Mixed-Methods Study on the Barriers and Facilitators of Telemedicine for Newborn Resuscitation. Telemed J E Health. (2018) 24:811-7. doi: 10.1089/tmj.2017.0182

27. Hollander JE, Carr BG. Virtually perfect? Telemedicine for covid-19. N Engl J Med. (2020) 382:1679-81. doi: 10.1056/NEJMp2003539

Conflict of Interest: The authors declare that the research was conducted in the absence of any commercial or financial relationships that could be construed as a potential conflict of interest.

Copyright (๑) 2021 Maddox, Albritton, Morse, Latendresse, Meek and Minton. This is an open-access article distributed under the terms of the Creative Commons Attribution License (CC BY). The use, distribution or reproduction in other forums is permitted, provided the original author(s) and the copyright owner(s) are credited and that the original publication in this journal is cited, in accordance with accepted academic practice. No use, distribution or reproduction is permitted which does not comply with these terms. 